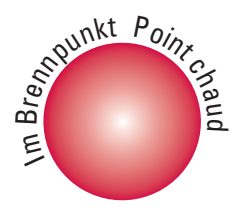

\title{
Cas cliniques Klinische Fälle
}

\section{Die mikrografische Chirurgie nach Mohs: Technik und Indikationen}

\section{Salomon, M. Adatto, A. Skaria}

Service de Dermatologie, Hôpitaux Universitaires de Genève, Genève, Suisse

\section{Einführung}

Das Prinzip der mikrografischen Chirurgie wurde von Dr. Frederic Mohs in den vierziger Jahren erarbeitet [1-5]. Diese Technik erlaubt es, kutane Karzinome unter Berücksichtigung zweier wesentlicher Gesichtspunkte zu behandeln: 1. maximale Sicherheit, dass die Exzision in toto erfolgt; 2. Verminderung der Exzisionsmarge und somit Einsparung von gesundem peritumoralem Gewebe.

\section{Konzept der mikrografischen Chirurgie}

Basalzellkarzinome (BZK) haben oft eine infraklinische Ausdehnung, welche an der Hautoberfläche nicht sichtbar ist (Eisberg-Phänomen, Abb. 1a, b). Diese Ausdehnung kann einerseits tiefer infiltrieren als klinisch erwartet (z.B. embryonale Fusionszonen) oder asymmetrisch sein, z.B. bei einem Rezidiv nach unvollständiger Exzision.

Angesichts eines rezidivierenden sklerodermiformen BZK des Nasenflügels (Abb. 1a) ist es für den Chirurgen schwierig, die korrekte Exzisionsmarge zu definieren. Die richtige Exzisionsmarge ist eine Funktion der klinischen Ausdehnung und der Histologie des Tumors, ein Kompromiss zwischen dem Risiko einer nicht totalen Exzision und der Einsparung von gesundem Gewebe. Je kleiner der Exzisionsdefekt, umso sicherer ist das funktionelle und ästhetische Resultat der Rekonstruktion. Die mikrografische Chirurgie erlaubt präzise, die Ausdehnung des Tumors zu erfassen. Das Karzinom in der Abbildung 1 wurde mikrografisch operiert und zeigte erst intraoperativ die Ausdehnung bis zur nasalen Mukosa und dem Nasenflügelrand, so dass der Exzisionsdefekt schliesslich durchgehend war (Abb. 1b).

\section{Das Prinzip der mikrografischen Chirurgie}

Im Unterschied zur herkömmlichen Schnellschnitttechnik, welche nur einen kleinen Teil des Exzisates untersucht, erlaubt es die mikrografische Chirurgie, den gesamten Schnittrand des Exzisates zu visualisieren und eventuelle Tumornester genau zu lokalisieren.
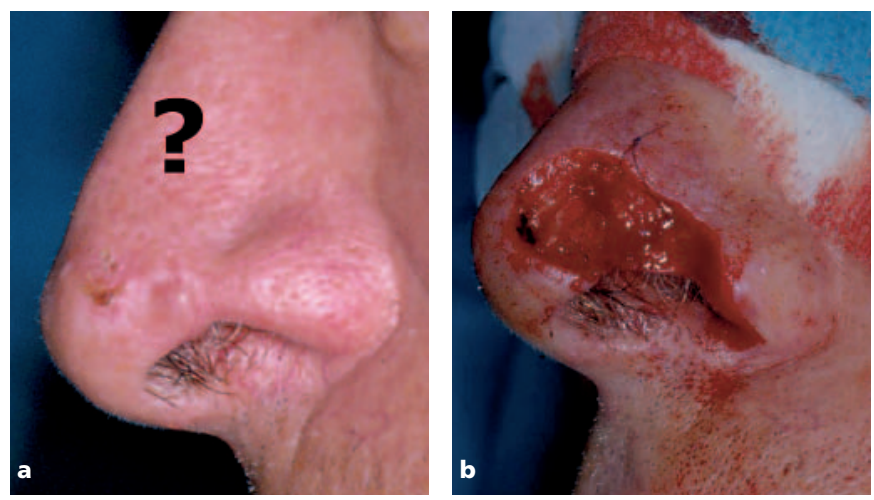

Abb. 1a, b. BZK: Eisberg-Phänomen.
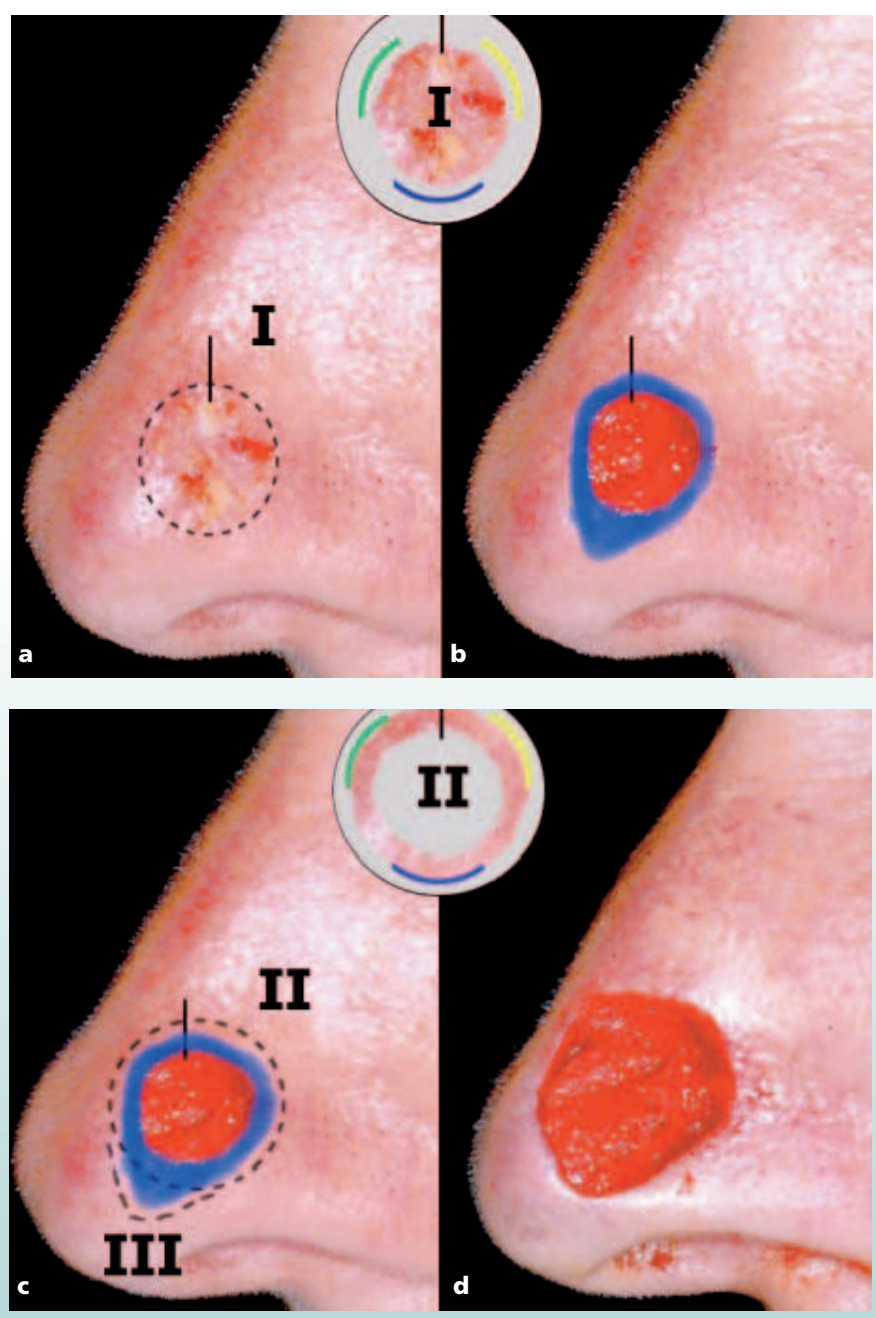

Abb. 2. Metatypisches BZK des Nasenflügels.

Die mikrografische Chirurgie ist ein Abwechseln zwischen chirurgischer Exzision und histologischer Untersuchung durch den behandelnden Chirurgen (und zugleich Histopathologen in einer Person), bis die gesamte Exzision des Karzinoms gewährleistet ist. Diese Technik gewährt eine minimale Exzision mit der maximalen Garantie der Vollständigkeit. Der Eingriff vollzieht sich unter Lokalanästhesie und ohne Hospitalisation. 


\section{Cas cliniques \\ Klinische Fälle}
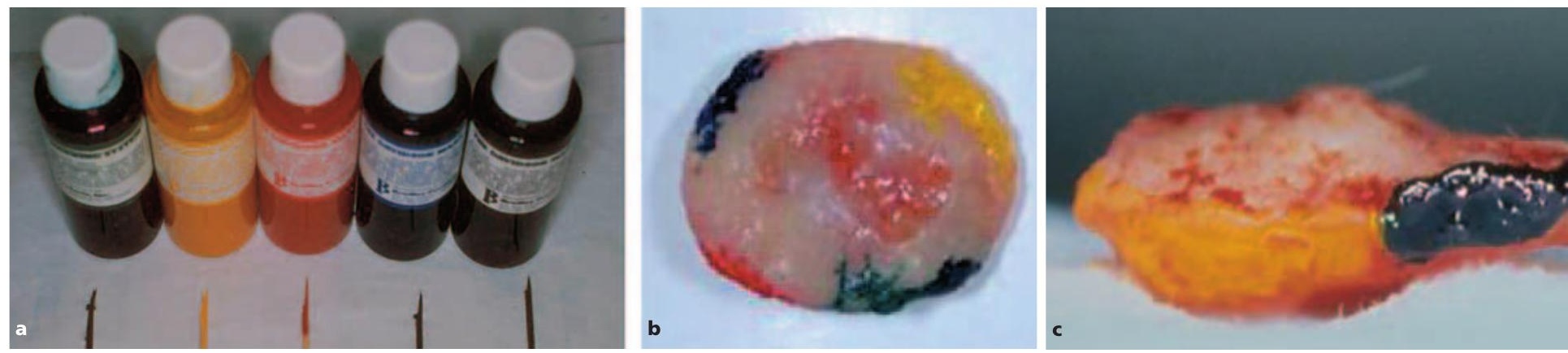

Abb. 3. Technik: Markierung der Exzisatränder.

Die Abbildung 2 zeigt ein metatypisches BZK des Nasenflügels. Der Tumor wird mit einer minimalen Sicherheitsmarge bezüglich des klinischen Bildes exzidiert (Abb. 2a). Eine Inzision bei 12.00 dient als Orientierung während des gesamten Verarbeitungsprozesses. Dieses Exzisat wird also nach dem histologischen Prinzip der mikrografischen Chirurgie verarbeitet (d.h. Horizontalschnitte im Gegensatz zu Vertikalschnitten der klassischen Histologie). In der Abbildung $2 \mathrm{~b}$ entspricht die blaue Farbe der Zone, wo der Tumor infiltriert, ohne klinisch sichtbar zu sein. Die erste Exzision (Medaillon I) ist noch vollständig im Tumor über die ganze Zirkumferenz hinweg.

Dann erfolgt eine Nachexzision der ganzen Zirkumferenz (Abb. 2c). Diese 2. Nachexzision (Medaillon II) wird mittels der gleichen histopathologischen Technik untersucht. Es persistiert eine tumorinfiltrierte Zone im unteren Segment (III), welche eine 3 . Nachexzision - in toto - bedingt.

\section{Technische Aspekte der mikrografischen Chirurgie}

Die Technik der mikrografischen Chirurgie basiert auf dem Prinzip der seriellen Horizontalschnitte von der Subkutis bis zur Epidermis der Histologie. Diese Technik erlaubt, den ganzen Schnittrand in der Tiefe und lateral des Exzisates zu beurteilen. Die Verarbeitung der Kryoschnitte und deren histologische Analyse dauern im Mittel 30-45 min in Abhängigkeit von der Grösse des Gewebestückes.

Unmittelbar nach der Exzision werden die Exzisatränder mittels verschiedenen Farben über die ganze Zirkumferenz markiert (Abb. 3a-c). Als Orientierung dient die Inzision bei 12.00 des Chirurgen (schwarzer Strich, Abb. 3b).

Das Gewebestück wird sodann auf einen Kryozylinder gelegt und im Kryomikrotom tiefgefroren (Abb. 4a). Danach wird das Gewebestück seriell horizontal von der Tiefe (Dermis, Hypodermis) bis zur Oberfläche (Epidermis) geschnitten. Ein Schnitt von 5-8 um Dicke wird alle 100-300 $\mu \mathrm{m}$ entnommen (Abb. 4b).

Am Ende werden 5-20, in Abhängigkeit der Dicke des Exzisates, Objektträger fixiert und gefärbt (Abb. 5). Diese Serienschnitte werden sodann durch den mikrografischen Chirurgen («Mohs surgeon») beurteilt.

Die Abbildung 6 zeigt das Karzinom (schwarze Farbe) inmitten des Exzisates. Das Karzinom ist auf dem ersten Schnitt
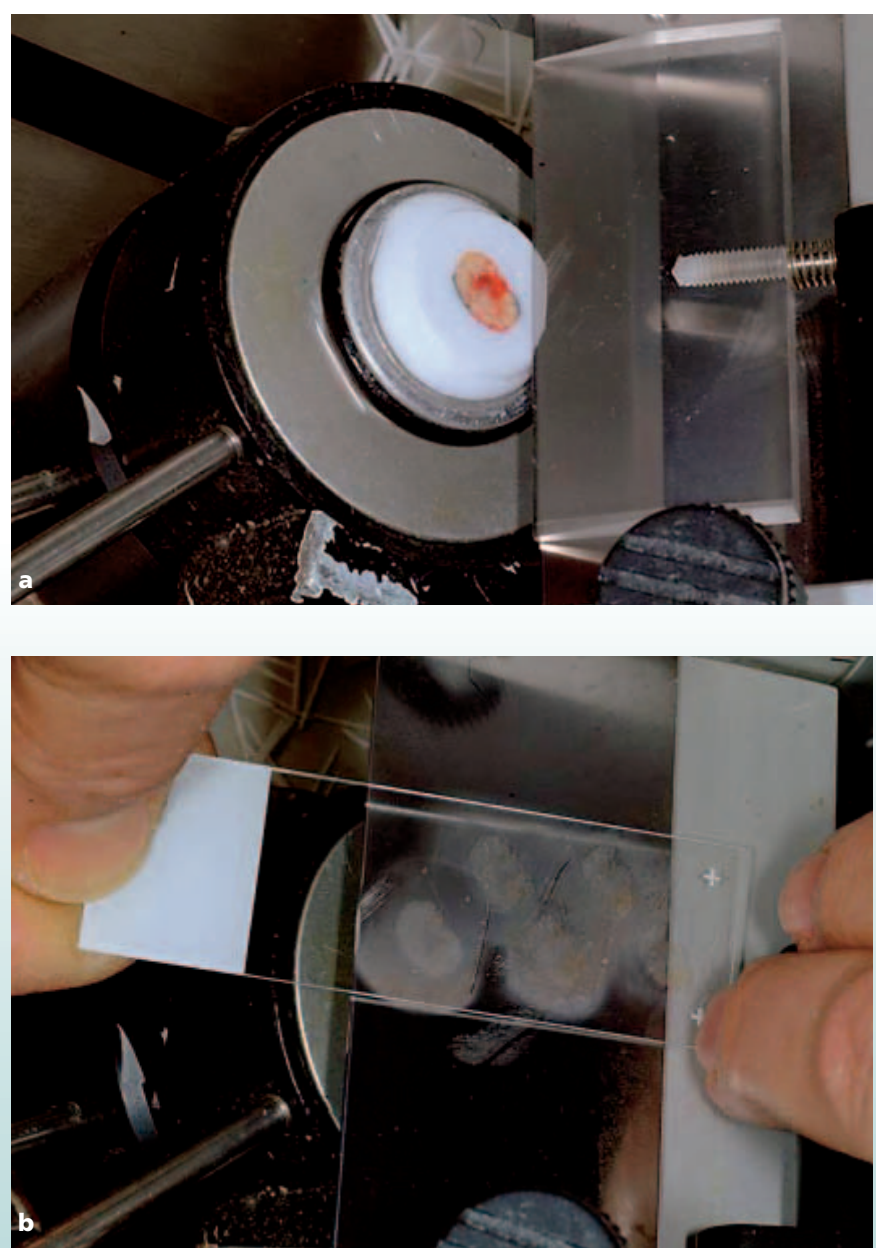

Abb. 4. Bearbeitung des Exzisats im Kryotom und Schnitte.

in der Tiefe und im Randbereich neben der Orientierungsinzision. Die Folgeuntersuchungen werden nach dem gleichen Prinzip verarbeitet, bis der Tumor vollständig exzidiert ist.

Im Allgemeinen vollführt der mikrografische Chirurg die Exzision, histopathologische Beurteilung und die Rekonstruktion in einer Person. Dies erlaubt einen besseren Überblick der verschiedenen Etappen, welche eine präzisere Nachexzision dank einer kombinierten chirurgisch-klinischen und 


\section{Cas cliniques \\ Klinische Fälle}

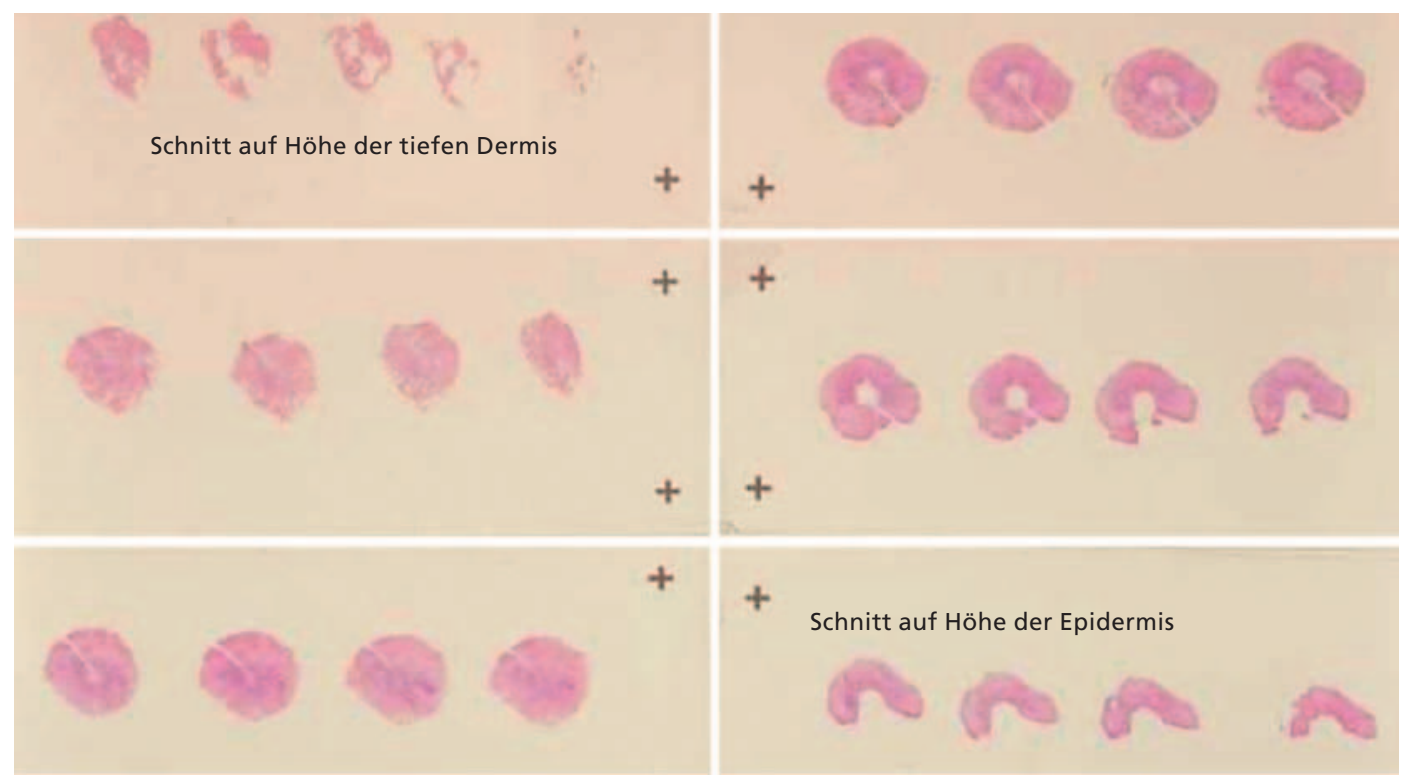

Abb. 5. Serienschnitte aus verschiedenen Tiefen.

histologischen Sicht ermöglicht. Die Fehlerquelle von Gewebefehlorientierung wird minimiert, was vor allem in dreidimensionalen Exzisionen (Ohr und Nase) wichtig sein kann. Der Gesamtablauf mit einfacher oder multiplen Exzisionen sowie Rekonstruktion nimmt 2-3 h in Anspruch, wobei noch die histopathologische Untersuchung dazugerechnet werden muss.

\section{Indikationen der mikrografischen Chirurgie}

Die BZK mit hohem Risiko («à haut risque») stellen den Grossteil der Indikationen dar. Die Auswahlkriterien der amerikanischen und europäischen mikrografisch chirurgischen Gesellschaften sind die folgenden.

Histopathologische Kriterien

- Sklerodermiforme, mikronoduläre, syringomatöse BZK

- BZK mit perineuraler Infiltration

- Mikrozystisch anexielles Karzinom

- Basalzell-Nävus-Syndrom

\section{Klinische Kriterien}

Die klinischen Kriterien berücksichtigen die Erfahrung und präoperative Beurteilung durch den behandelnden Arzt.

- BZK mit einer klinischen Ausdehnung, welche nach der Exzision eine Rekonstruktion mittels Lappenplasik benötigen. In dieser Situation kann die Rekonstruktion bei unvollständiger Exzision zur Versetzung von Tumornestern führen. Diese befinden sich in der Folge abseits vom primären Tumorherd und erschweren die Lokalisierung und genaue Beurteilung der Ausdehnung bei einer eventuellen Nachexzision oder eines Rezidives.

- BZK der Nase, Augenlider, Augenbrauen oder perioral. In diesen Regionen ist es wichtig, ein Maximum an gesundem Gewebe zu erhalten.

- BZK-Rezidiv (Abb. 7) nach vorangehender Behandlung mittels Chirurgie, Radiotherapie oder anderen Therapien.
Beispiel eines rezidivierenden BZK nach zwei chirurgischen Exzisionen mit histologischem Nachweis einer perineuralen Infiltration des supraorbitalen Nervs.

- BZK der embryonalen Fusionszonen (Abb. 8). Beispiel eines BZK, welches den Nasensulkus infiltriert. Klinisch war es unmöglich, die Ausdehnung des Tumors zu beurteilen. Eine totale Exzision war nur dank der mikrografischen Chirurgie möglich.

- BZK, welche in der Tiefe nicht vollständig exzidiert wurden.

- Immunsupprimierte Patienten.

Andere Indikationen der mikrografischen Chirurgie sind nicht vollständig exzidierte oder tief infiltrierende und/oder perineural infiltrierende Spinalzellkarzinome, Dermatofibro-

Abb. 6. Schema der Exzidierung.

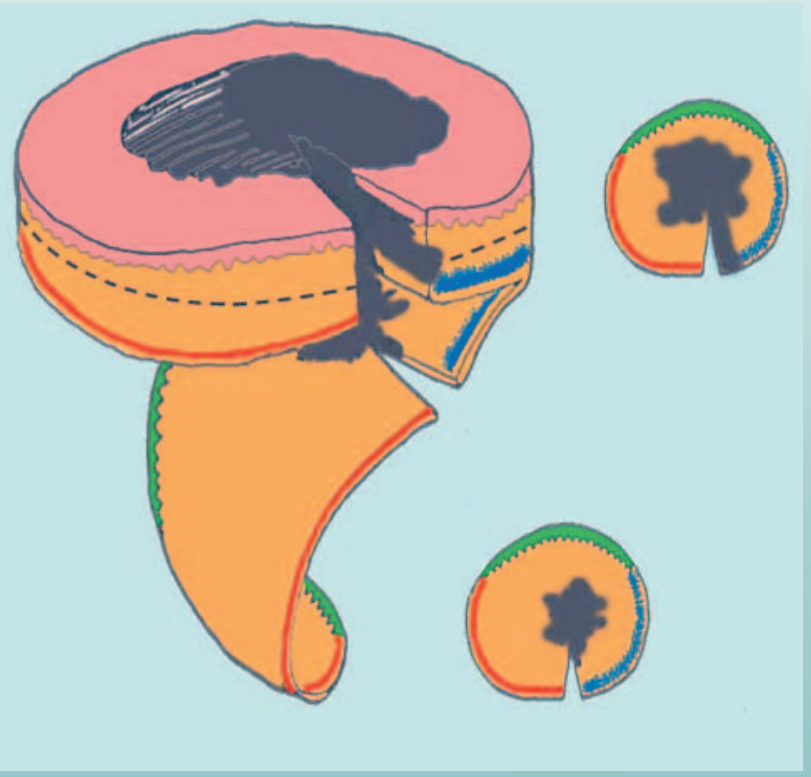

Avec les compliments des éditions S. Karger, Bâle Mit den besten Empfehlungen des Verlags S. Karger, Basel 


\section{Cas cliniques \\ Klinische Fälle}
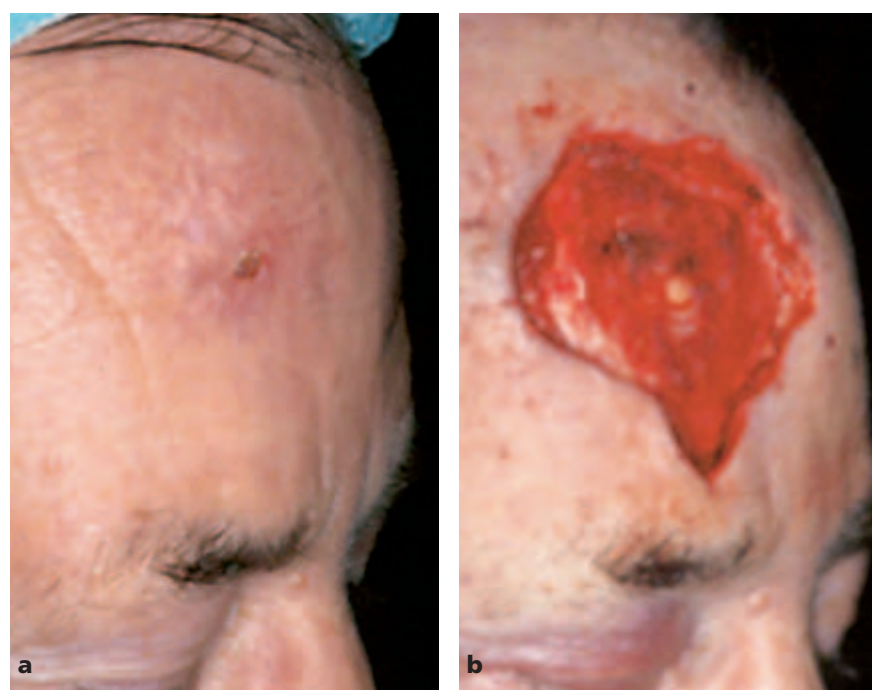

Abb. 7. BZK-Rezidiv.
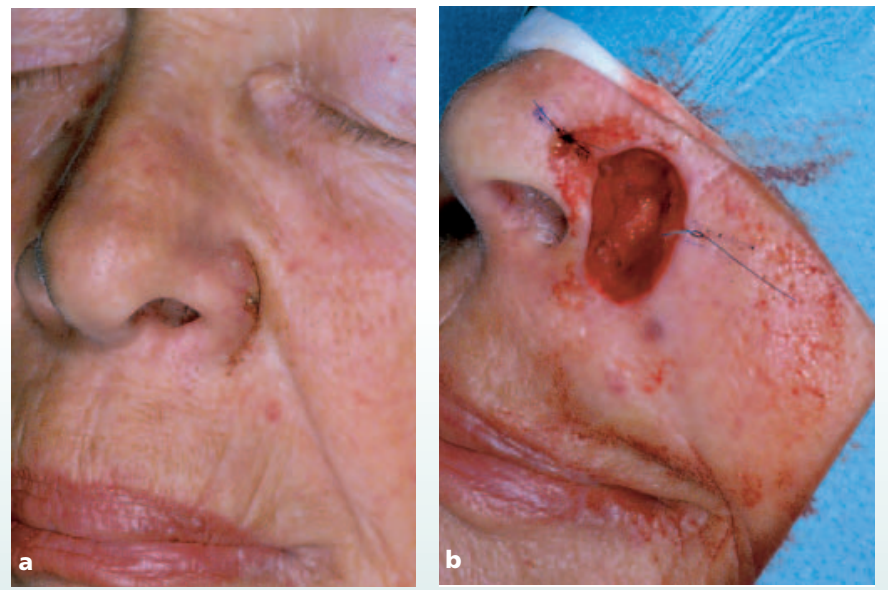

Abb. 8a, b. BZK der embryonalen Fusionszonen.

sarkome und gewisse Lentigines malignae. Für die zwei letzteren Indikationen ist eine Formolfixierung und Paraffineinbettung empfohlen, wobei die horizontale Schnitttechnik modifiziert wird.

Es sollte klar sein, dass die konventionelle histologische Untersuchung keine vollständige Analyse der Schnittränder garantiert; dies erhöht das Risiko einer falsch negativen Untersuchung und somit eines Rezidives.

\section{Schlussfolgerung}

Die mikrografische Chirurgie verbindet die mikroskopische Analyse des gesamten Exzisates mit einer exakten Kartografie, welche es erlaubt, den Tumor dreidimensional genau zu lokalisieren. Die Verarbeitung wird mittels Gefrierschnitttechnik durchgeführt, welche eine Beurteilung unmittelbar nach der Entnahme ermöglicht. Diese Technik ermöglicht eine Verminderung der Exzisionsmarge und sichert eine voll- ständige Exzision des Tumors. Die Indikationen der mikrografischen Chirurgie sind klar etabliert und sollten in Zukunft ihren Platz in der Behandlung der kutanen Karzinome finden.

\section{Literatur}

1 Smeets NWJ, Kuijpers DIM, Nelemans P, et al: Mohs micrographic surgery for treatment of basal cell carcinoma of the face: results of a retrospective study and comparison with literature results. $\mathrm{Br}$ J Dermatol 2004;151:141-147.

2 Sei JF, Chaussade V, Zimmermann U, Tchakerian A, Clerici T, Franc B, Saiag P: Historique, principes, analyse critique de l'efficacité et indications de la chirurgie micrographique de Mohs. Ann Dermatol Vénéréol 2004;131:173-182.

3 Essers BAB, Dirksen CD, Nieman FHM, Smeets NWJ, Krekels GAM, Prins MH, Martino Neumann HAM: Cost-effectiveness of Mohs micrographic surgery versus surgical excision for basal cell carcinoma of the face. Arch Dermatol 2006;142:187-194.

4 Chren MM: Determining the value of surgical therapies for basal cell carcinoma. Arch Dermatol 2006;142:231-232.

5 Bath-Hextall F, Bong J, Perkins W, Williams H: Interventions for basal cell carcinoma of the skin: systematic review. BMJ 2004; 329:705.

La version française de cet article paraîtra dans un de nos prochains numéros. 